\title{
Local Evolution of the Elastocaloric Effect in TiNi-Based Films
}

\author{
H. Ossmer ${ }^{1}$ - C. Chluba ${ }^{2}$ M. Gueltig ${ }^{1} \cdot$ E. Quandt ${ }^{2} \cdot$ M. Kohl ${ }^{1}$
}

Published online: 16 May 2015

(C) ASM International 2015

\begin{abstract}
Strain and temperature profiles of magnetronsputtered ferroelastic TiNi-based films of $20 \mu \mathrm{m}$ thickness are investigated during tensile load cycling with respect to strain, strain rate, and cycle number in order to assess their potential for elastocaloric cooling. Two different ferroelastic film specimens are considered, binary $\mathrm{TiNi}$ and quaternary TiNiCuCo films, which strongly differ regarding their phase transformation hysteresis and fatigue behavior. In situ digital image correlation and infrared thermography measurements reveal a correlated response of strain and temperature bands that is determined by mesoscale stress and temperature fields on the kinetics of phase transformation. In the case of binary TiNi films, this response is also strongly affected by cycling-induced fatigue causing vanishing band formation and decreasing elastocaloric effect size. In contrast, TiNiCuCo films show negligible fatigue and retain the local characteristics of the elastocaloric effect. Compared to TiNi films, they exhibit not only a reduced temperature change, but also a reduced work input for pseudoelastic cycling resulting in an improved material's coefficient of performance of 15 .
\end{abstract}

Keywords Shape memory films · TiNi .

Pseudoelasticity · Elastocaloric effect

H. Ossmer

hinnerk.ossmer@kit.edu

1 Institute of Microstructure Technology, Karlsruhe Institute of Technology, P.O. Box 3640, 76021 Karlsruhe, Germany

2 Institute for Material Science, University of Kiel, 24143 Kiel, Germany

\section{Introduction}

In recent years, research on caloric effects in solid state devices encounters considerable interest as they promise novel applications in cooling and heat pumping with potentially high energy efficiency and low environmental impact [1-3]. Elastocaloric cooling in pseudoelastic shape memory alloys (SMAs) is caused by the stress-induced transformation from austenite (A) to martensite (M) [4], which is associated with a large latent heat up to $31 \mathrm{~J} \mathrm{~g}^{-1}$ in the case of binary TiNi [5]. The corresponding direct temperature change $\Delta T$ can reach up to $40 \mathrm{~K}$ [6]. In contrast to other caloric effects relying on high magnetic or electric fields $[1,2,7]$, the driving force for elastocaloric cooling is mechanical stress or strain. This approach is highly attractive for miniature cooling applications, such as active cooling in microelectronics, bio-medicine, and chemical analytics, e.g., local cooling of biological tissue, rapid blood cooling in microsurgery, temperature stabilization of lab-on-chip systems [8, 9]. The high surface-tovolume ratio inherent to films is in this regard advantageous, since it promises large heat transfer rates and, concomitant, high cycling frequencies.

For the development of an elastocaloric film device, it is essential to understand and control the evolution of strain and temperature profiles during cyclic operation. Major challenges are related to tailoring of effect size $(\Delta T)$ and minimizing fatigue effects. Recent investigations on magnetron-sputtered binary TiNi films during load cycling reveal a large effect size similar to bulk [10]. However, these films tend to suffer from functional fatigue leading to plastic deformation and accumulation of remanent strain as well as decrease of the elastocaloric temperature change [11]. Different strategies may be pursued to cope with this issue and to develop ferroelastic SMA film materials with 
improved intrinsic and extrinsic properties. One option is the development of textured films. Textured cold-rolled TiNi foils of 30- $\mu \mathrm{m}$ thickness have been shown to exhibit improved fatigue resistance without degradation of the elastocaloric effect [12]. Another option is alloying of third and fourth elements. Combinatorial investigations by systematic screening in ternary and quaternary thin film systems have been successfully applied to identify alloys with hysteresis close to zero and improved reversibility of phase transformation [13], which is attributed to the higher crystallographic compatibility between parent and product phase. Ternary TiNiCu films, for instance, show stressstrain characteristics with narrow hysteresis and improved fatigue properties [11]. Recent studies on alloying of fourth elements have shown that Co addition provides a way to lower the transformation temperatures while maintaining high transformation enthalpies [14].

In this paper, we present an analysis of time-resolved strain and temperature profiles on the mesoscopic (micrometer) length scale in magnetron-sputtered $\mathrm{TiNi}$ and TiNiCuCo films during load cycling. By adding of Co, quaternary TiNiCuCo films have been developed with reduced austenitic finish temperature $A_{\mathrm{f}}$ showing ferroelastic performance at room temperature with high cyclic stability. The effects of strain, strain rate, and functional fatigue in the films on the local evolution of the elastocaloric effect will be addressed. The potential of TiNi-based films for small-scale applications will be discussed.

\section{Experimental}

Freestanding film samples are produced by DC magnetron sputtering onto a glass substrate with pre-structured sacrificial copper layer [15]. For tensile tests, the samples are structured in stripes of $1.75-\mathrm{mm}$ width and $15-\mathrm{mm}$ length. TiNi films are fabricated using a $4 " \mathrm{Ti}_{53.2} \mathrm{Ni}_{46.8}$ at. \% alloy target. TiNiCuCo film samples are produced by co-sputtering of an $8 " \mathrm{Ti}_{56} \mathrm{Ni}_{29} \mathrm{Cu}_{15}$ at. \% alloy target and a Co target in multilayers of $35-$ and $\sim 1-\mathrm{nm}$ thickness, respectively. Homogeneity is established through annealing. Additionally, a 4" $\mathrm{Ti}_{58} \mathrm{Ni}_{24.5} \mathrm{Cu}_{14.7} \mathrm{Co}_{2.3}$ alloy target with an optimized composition is used. Co is chosen as a fourth element to shift the phase transformation temperature to be near room temperature [14]. The sputtering power is $300 \mathrm{~W}$ and the Ar flow rate $20 \mathrm{sccm}$ at $2 \times 10^{-3} \mathrm{mbar}$ during sputter deposition. Film thicknesses have been adjusted to about $20 \mu \mathrm{m}$. After sputtering, the films are released by selective wet-etching of the sacrificial $\mathrm{Cu}$ layer. Film compositions are determined by energy dispersive $\mathrm{X}$-ray analysis (EDX) to be $\mathrm{Ti}_{49.6} \mathrm{Ni}_{50.4}$ for the binary films, $\mathrm{Ti}_{54.7} \mathrm{Ni}_{30.7} \mathrm{Cu}_{12.3} \mathrm{Co}_{2.3}$ for the multilayer prepared films and $\mathrm{Ti}_{55} \mathrm{Ni}_{29.6} \mathrm{Cu}_{12.6} \mathrm{Co}_{2.8}$ for the single target films. The as-deposited freestanding films are amorphous and, thus, require a heat treatment to crystallize the films and to adjust their phase transformation properties. The heat treatment is adjusted in a way to establish the austenite finish temperature close below $22{ }^{\circ} \mathrm{C}$ to enable superelastic tests at room temperature. For the TiNi films, the heat treatment procedure comprises two-step rapid thermal annealing at $650{ }^{\circ} \mathrm{C}$ for $10 \mathrm{~min}$ and at $450{ }^{\circ} \mathrm{C}$ for $10 \mathrm{~min}$. A single heating step is performed at 700 and $780{ }^{\circ} \mathrm{C}$ for $15 \mathrm{~min}$ for the $\mathrm{Ti}_{54.7} \mathrm{Ni}_{30.7} \mathrm{Cu}_{12.3} \mathrm{Co}_{2.3}$ and the $\mathrm{Ti}_{55} \mathrm{Ni}_{29.6} \mathrm{Cu}_{12.6} \mathrm{Co}_{2.8}$ film, respectively.

The phase transformation temperatures, latent heat, and heat capacity are determined by differential scanning calorimetry (DSC) under nitrogen inert gas flow of $10 \mathrm{ml} \mathrm{s}^{-1}$ at a heating rate of $10 \mathrm{~K} \mathrm{~min}^{-1}$. Measurements of specific heat capacity $c_{\mathrm{p}}$ are performed at a heating rate of $20 \mathrm{~K} \mathrm{~min}^{-1}$ using a synthetic sapphire standard. Complementary temperature-dependent electrical resistance measurements are conducted in a thermostat under vacuum of about $2 \times 10^{-2}$ mbar. Quasi-static conditions are maintained by awaiting stationary measurement signals at each data point before data acquisition.

Uniaxial tensile tests are performed at room temperature in air. A schematic of the test setup is shown in Fig. 1. The tensile testing machine is equipped with a $50 \mathrm{~N}$ force sensor having a resolution of $0.25 \mathrm{~N}$. The resolution of the displacement sensor is $0.25 \mu \mathrm{m}$. Test samples are attached to small alumina plates with two-component adhesive, which are then fixed to the clamping of the machine by screws. Engineering strains are calculated from the crossbeam position $L(t)$ of the tensile test machine and the initial

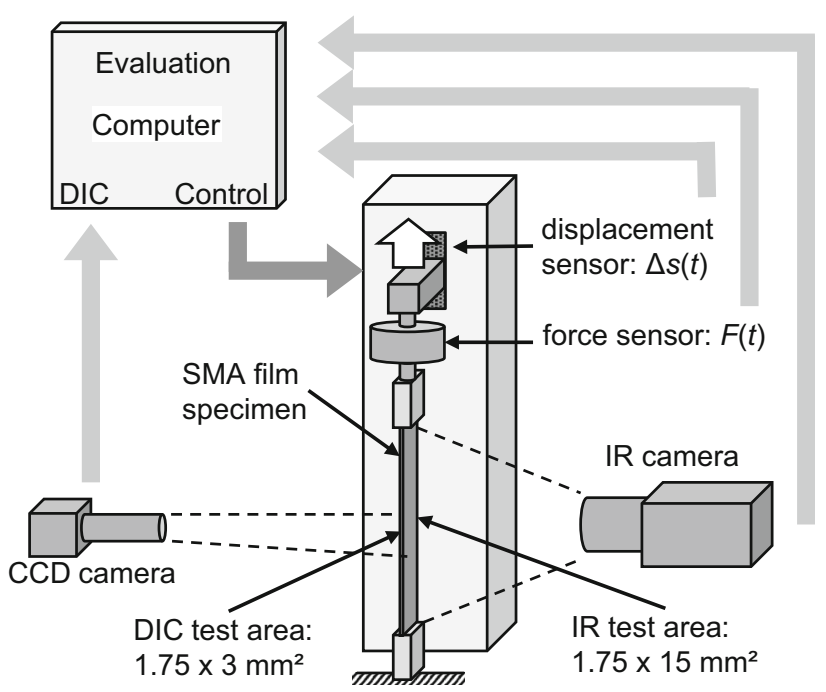

Fig. 1 Schematic of tensile test setup. Infrared and optical images are recorded in situ during tensile load cycling to determine temperature profiles and local strain fields by digital image correlation, respectively 
sample length $L_{0}$ according to $\varepsilon=\left(L(t)-L_{0}\right) / L_{0}$. Cyclic load programs in strain-control mode are performed at various strain rates between $10^{-4}$ and $2 \mathrm{~s}^{-1}$.

A typical load cycle is operated in the following way: (a) First, a tensile pre-load of $25 \mathrm{MPa}$ is applied to avoid buckling of the sample. (b) The strain is increased at constant strain rate until the maximum strain $\varepsilon_{\max }$ is reached, which is limited to keep slightly below the end of the pseudoelastic plateau. (c) The maximum strain is maintained for $10 \mathrm{~s}$ to allow for heat release. (d) The strain is decreased at constant strain rate until the tensile pre-load of $25 \mathrm{MPa}$ is reached. (e) The pre-load is maintained for $10 \mathrm{~s}$ to allow for heat absorption.

During load cycling, the temperature profile of the sample is monitored in situ by an infrared camera. Samples are covered with a thin layer of graphite to improve thermal emissivity. Images are acquired with a frame rate of up to $100 \mathrm{~Hz}$ at a spatial resolution of $25 \mu \mathrm{m}$. Optical images of $2 \times 3 \mathrm{~mm}^{2}$ test areas are recorded in situ with a spatial resolution of $2.5 \mu \mathrm{m}$ per pixel by a CCD camera to evaluate local strain profiles using the digital image correlation (DIC) technique [16]. For DIC, a speckle pattern is created by spraying a thin layer of graphite, as the surface of sputter-deposited samples is flat and featureless.

\section{Phase Transformation Properties}

Temperature-dependent electrical resistance and DSC curves are shown in Figs. 2 and 3 for both materials. The electrical resistance curve of TiNi exhibits the characteristic anomaly of a two-stage transformation [17]. Upon

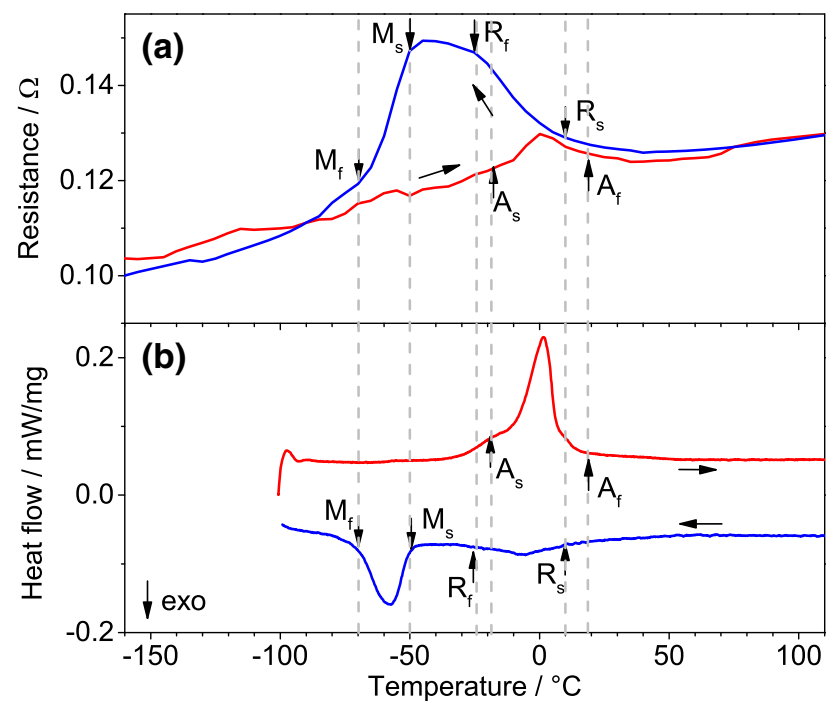

Fig. 2 a Electrical resistance and b DSC measurements for the $\mathrm{Ti}_{49.6} \mathrm{Ni}_{50.4}$ film. The martensitic transformation proceeds in two steps via the intermediate R-phase. Phase transformation temperatures are indicated

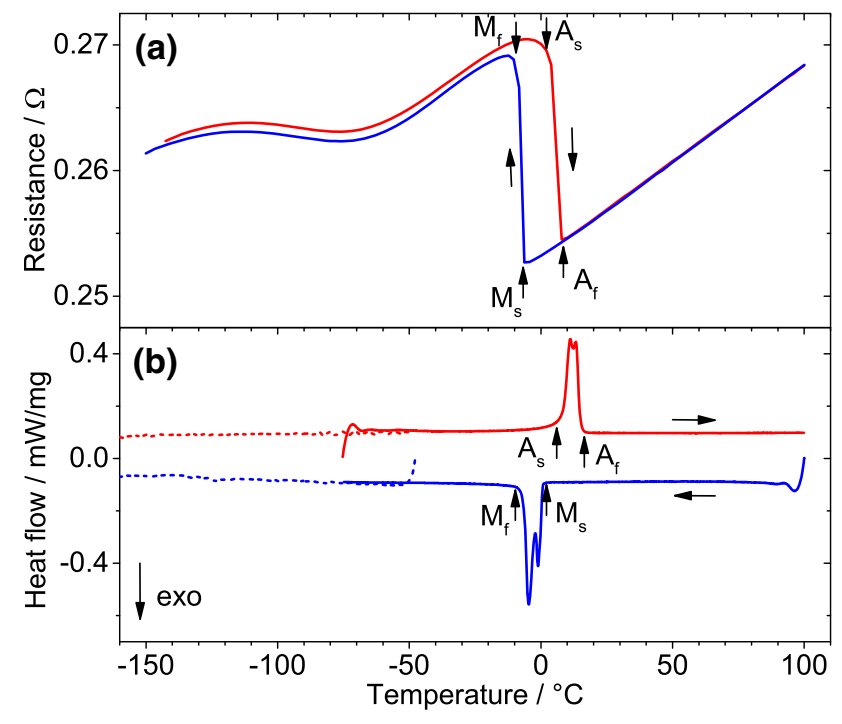

Fig. 3 a Electrical resistance and b DSC measurements for the $\mathrm{Ti}_{54.7} \mathrm{Ni}_{30.7} \mathrm{Cu}_{12.3} \mathrm{Co}_{2.3}$ film showing a single-step martensitic transformation. Phase transformation temperatures are indicated

cooling, the increasing slope indicates the start of the intermediate R-phase transformation. The subsequent drop of electrical resistance is due to the martensitic transformation. Upon heating, the corresponding reverse transformations occur. Phase transformation temperatures are determined by applying the tangential method and are summarized in Table 1. The observed transitions correlate well with the DSC results. Here, the R-phase transformation upon cooling is reflected by a small peak at about $-5{ }^{\circ} \mathrm{C}$, while the large peak near $-60{ }^{\circ} \mathrm{C}$ is due to the martensitic transformation. Upon heating, a main peak at about $0{ }^{\circ} \mathrm{C}$ with a shoulder at $-20^{\circ} \mathrm{C}$ is observed reflecting the reverse transformations from martensite via $\mathrm{R}$-phase to austenite. The latent heat of the full transformation obtained by integration of the peak area is determined to be $18.9 \mathrm{~J} \mathrm{~g}^{-1}$.

In comparison, quaternary TiNiCuCo samples show a single-stage phase transformation. Electrical resistance curves upon heating and cooling show sharp steps in the transformation temperature range of -10 to $15^{\circ} \mathrm{C}$, which correlate well with the DSC results. In electrical resistance, also a small broad hump occurs at $-160^{\circ} \mathrm{C}$, which has no counterpart in the DSC curve. The double peaks seen in the DSC curve (Fig. 2b) are attributed to slight inhomogeneity of the sample, which also leads to broader transitions in the DSC, compared to the resistance measurement. The observed DSC peaks are very sharp showing a full width at half maximum of less than $7 \mathrm{~K}$. This is in line with the small hysteresis of the transition in the electrical resistance curves. Similar results are found in the literature on ternary $\mathrm{TiNiCu}$ films, which, however, transform above room temperature [18]. 
Table 1 Material properties of sputter-deposited $\mathrm{Ti}_{49.6} \mathrm{Ni}_{50.4}$ and $\mathrm{Ti}_{54.7} \mathrm{Ni}_{30.7} \mathrm{Cu}_{12.3} \mathrm{Co}_{2.3}$ film samples

\begin{tabular}{lll}
\hline & $\mathrm{Ti}_{49.6} \mathrm{Ni}_{50.4}-\mathrm{film}$ & $\mathrm{Ti}_{54.7} \mathrm{Ni}_{30.7} \mathrm{Cu}_{12.3} \mathrm{Co}_{2.3}-\mathrm{film}$ \\
\hline Start (s) and finish (f) temperatures & & 3,8 (DSC: 8, 14) \\
Austenite (A): $A_{\mathrm{s}}, A_{\mathrm{f}}\left({ }^{\circ} \mathrm{C}\right)$ & $-10,20$ & - \\
R-phase $(\mathrm{R}): R_{\mathrm{s}}, R_{\mathrm{f}}\left({ }^{\circ} \mathrm{C}\right)$ & $11,-23$ & $-6,-9$ (DSC: $1,-6)$ \\
Martensite $(\mathrm{M}): M_{\mathrm{s}}, M_{\mathrm{f}}\left({ }^{\circ} \mathrm{C}\right)$ & $-49,-70$ & 13.7 \\
Latent heat: $L\left(\mathrm{~J} \mathrm{~g}{ }^{-1}\right)$ & 18.9 & 0.42 \\
Specific heat capacity: $c_{\mathrm{p}}\left(\mathrm{J} \mathrm{g}^{-1} \mathrm{~K}^{-1}\right)$ & 0.40 & 1.0 \\
Work: $\Delta W\left(\mathrm{~J} \mathrm{~m}{ }^{-3}\right)($ closed pseudoelastic cycle, quasi-static) & 7.3 & 300 \\
Plateau stress $\sigma_{\text {crit }}(\mathrm{MPa})$ & 400 & \\
\hline
\end{tabular}

Phase transformation temperatures are determined from electrical resistance and DSC measurement by the tangential method. The work and plateau stress are determined by tensile measurements under quasi-static conditions

An overview on the phase transformation properties of the investigated samples is given in Table 1. Compared to $\mathrm{TiNi}$, the TiNiCuCo samples reveal a lower latent heat of $14 \mathrm{~J} \mathrm{~g}^{-1}$. The specific heat capacities $c_{\mathrm{p}}$, obtained from DSC measurements with a sapphire standard, are similar for both materials. Concerning applications in elastocaloric cooling, tailoring of the material parameters: latent heat, thermal hysteresis, and austenite finish temperature $A_{\mathrm{f}}$, is important. The latent heat determines the elastocaloric effect size, which is very large in binary TiNi. However, TiNi films exhibit also large hysteresis and considerable fatigue during load cycling. It has been shown that alloys showing narrow hysteresis also exhibit improved reversibility of phase transformation [13]. Such conditions can be reached in TiNiCu alloys, for which the middle eigenvalue of the transformation stretch tensor is close to 1.0 [19]. By addition of $\mathrm{Co}$, the phase transformation can be tuned to below room temperature, while the narrow transformation hysteresis and pseudoelastic cycling stability are maintained. Thus, TiNiCuCo films appear to be a promising material system for elastocaloric cooling. As a drawback, TiNiCuCo samples show reduced latent heat. However, for elastocaloric cooling, the ratio of latent heat $L$ and work required for load cycling $\Delta W$, namely the coefficient of performance COP $(=L / \Delta W)$, is an important figure-of-merit. Therefore, reduced latent heat could be accepted, if the work input $\Delta W$ is reduced in a similar way, $\Delta W$ depending on the hysteresis width and on the difference of austenite finish temperature $A_{\mathrm{f}}$ and operation temperature of the cooling device. The mechanical performance during load cycling will be investigated in the following.

\section{Local Mechanical Performance}

Cyclic tensile experiments are performed at various strain rates. The maximum engineering strain $\varepsilon_{\max }$ is determined for each sample by initial quasi-static tensile loading until the end of the pseudoelastic plateau is reached. As a result, we obtain about $6 \%$ for TiNi films and $2.75 \%$ for TiNi$\mathrm{CuCo}$ films. Figure 4 shows typical stress-strain characteristics at a strain rate of $0.02 \mathrm{~s}^{-1}$. In the first cycle, the shape of the curves is very similar for both types of films. Pronounced stress plateaus are observed due to stress-induced forward and reverse martensitic transformation. Upon loading, the stress plateau is about one-third higher
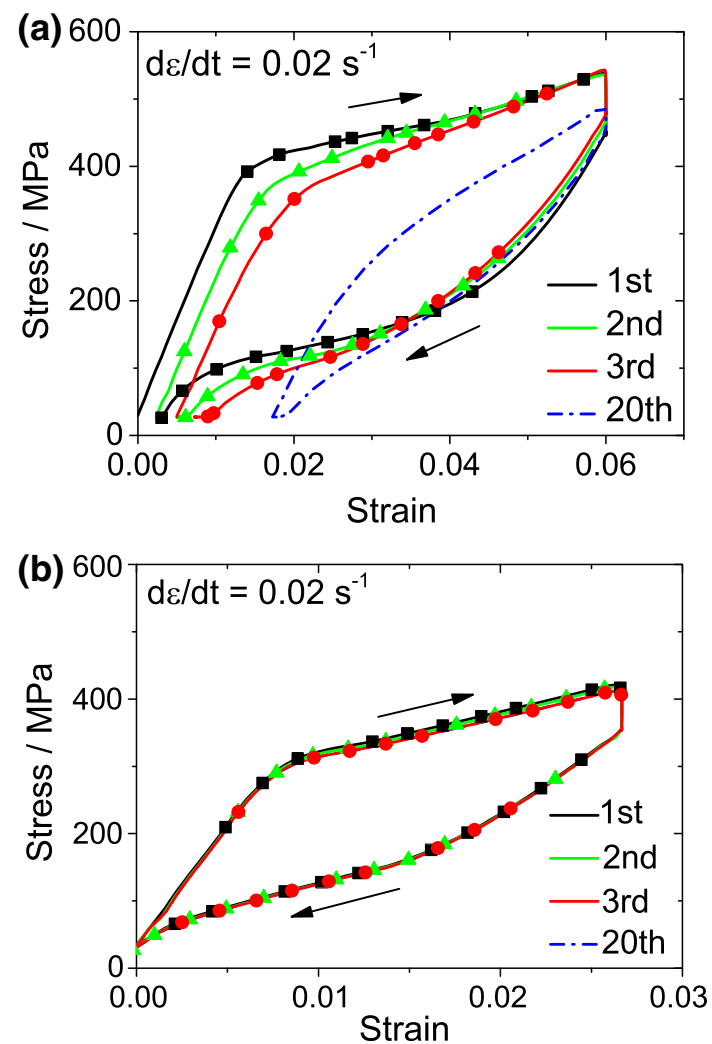

Fig. 4 Stress-strain characteristics of a tensile test sample of $\mathrm{Ti}_{49.6} \mathrm{Ni}_{50.4}$ (a) and $\mathrm{Ti}_{54.7} \mathrm{Ni}_{30.7} \mathrm{Cu}_{12.3} \mathrm{Co}_{2.3}$ film (b) at a strain rate of $0.02 \mathrm{~s}^{-1}$ for several load cycles as indicated. Symbols indicate stress-strain values where strain maps have been determined by DIC 
for the TiNi sample (400 MPa), compared to the TiNiCuCo sample (300 MPa). This is in line with the DSC results discussed above, which reveal that TiNi has lower transformation temperatures than TiNiCuCo. The difference of stress levels between loading and unloading correlates with the transformation hysteresis observed in the temperaturedependent experiments in Sect. 3. In particular, TiNiCuCo films exhibit a smaller hysteresis compared to the TiNi films.

During tensile testing, greyscale images of a $2 \times 3 \mathrm{~mm}^{2}$ test area are acquired in situ at high resolution by a CCD camera and evaluated using the DIC technique to determine local strain profiles at the mesoscale. In the following, the mechanical performance is discussed with respect to the dependence on strain, strain rate, and cycle number.

\section{Strain Dependence}

Local strain profiles of a $\mathrm{TiNi}$ and $\mathrm{TiNiCuCo}$ sample are shown in Figs. 5 and 6, respectively, for the first three loading cycles. The stress-strain values corresponding to the acquired strain maps are marked by symbols in Fig. 4. Initially, the local strain increases homogeneously and linearly with the engineering strain. At a critical stress $\sigma_{\text {crit }}$, one or several strain bands form. These so-called Lüders bands [20] are very fine in the initial stage. Sharp phase boundaries separate fully transformed regions of oriented martensite showing maximum strain from untransformed regions. The strain bands are oriented by an angle of about $55^{\circ}$ with respect to the loading direction and partly cross each other. For increasing strain, existing bands grow in size by propagation of phase boundaries and additional bands may form until the whole sample is transformed. During unloading, the reverse process occurs. Strain bands decrease in size and strain bands disappear until the initial strain-free state is reached. The observation of an abrupt and highly localized course of stress-induced phase transformation is in line with previous research on TiNi films [10], foils [12, 21], wires [22], tubes [23, 24], and belt-like samples [6]. It is important to note that this behavior strongly depends on the strain rate and fatigue behavior of the sample, as will be discussed in the following.

\section{Strain-Rate Dependence}

For increasing strain rate, locally released and absorbed latent heat accumulates due to limited heat transfer, which causes self-heating and self-cooling during loading and unloading, respectively. These effects are negligible at low strain rates of $10^{-4} \mathrm{~s}^{-1}$ allowing for quasi-stationary conditions. In this case, only one strain band at each end of the tensile test sample forms and evolves [10]. Corresponding stress-strain characteristics show almost horizontal pseudoelastic stress plateaus up to $6 \%$ strain. For increasing strain rate, discontinuous formation of many strain bands is favored compared to continuous progression of one strain band. Upon loading, this effect results from the local increase of temperature by self-heating, which impedes the continuous progression of phase transformation as will be discussed in more detail below in Sect. 5. The higher temperature raises the critical stress for phase transformation and, thus, stabilizes austenite. As a consequence, stress-strain characteristics show a finite slope of the stress plateau (see, e.g., Figure 4) [10, 25]. Similarly, the
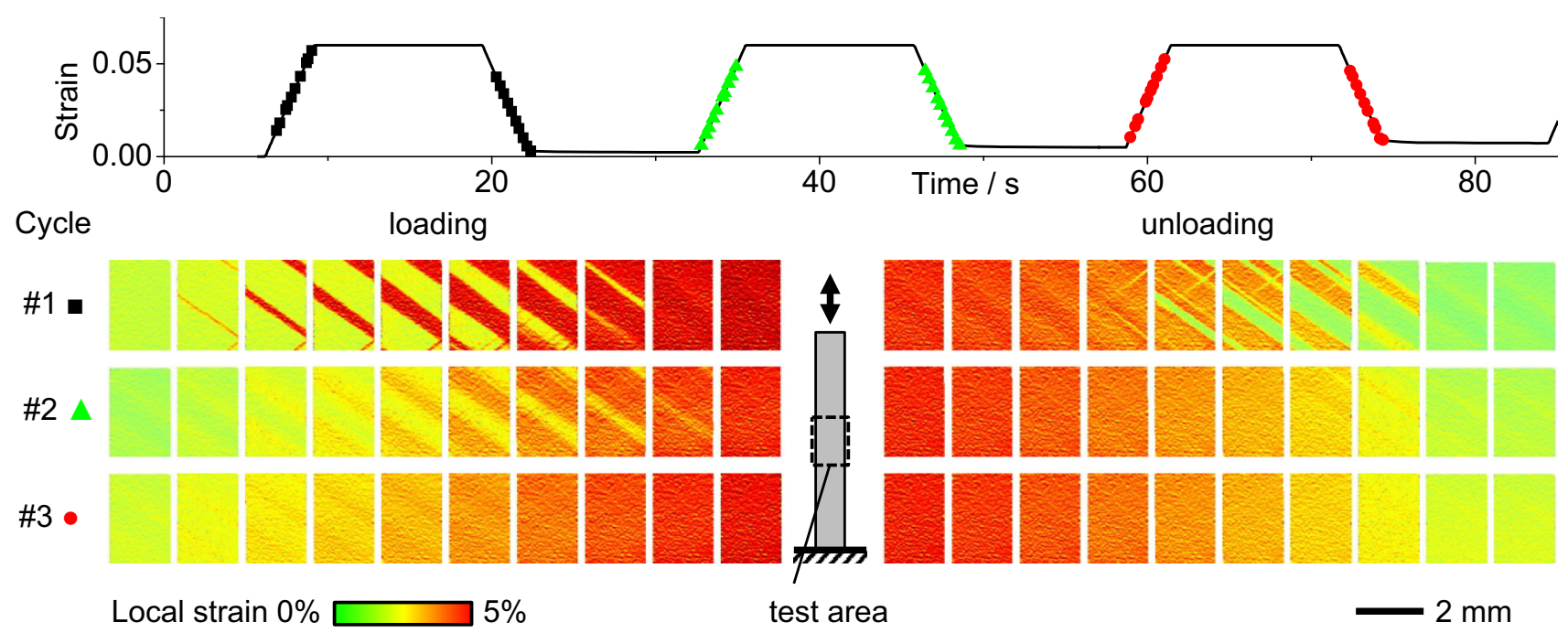

Fig. 5 Time series of local strain maps of a $\mathrm{Ti}_{49.6} \mathrm{Ni}_{50.4}$ sample obtained by DIC in a test area $\left(2 \times 3 \mathrm{~mm}^{2}\right)$ from three successive load cycles at a strain rate of $0.02 \mathrm{~s}^{-1}$. The points of image acquisition are marked by symbols in the engineering strain-time diagram above, as well as in the stress-strain characteristic of Fig. 4a 


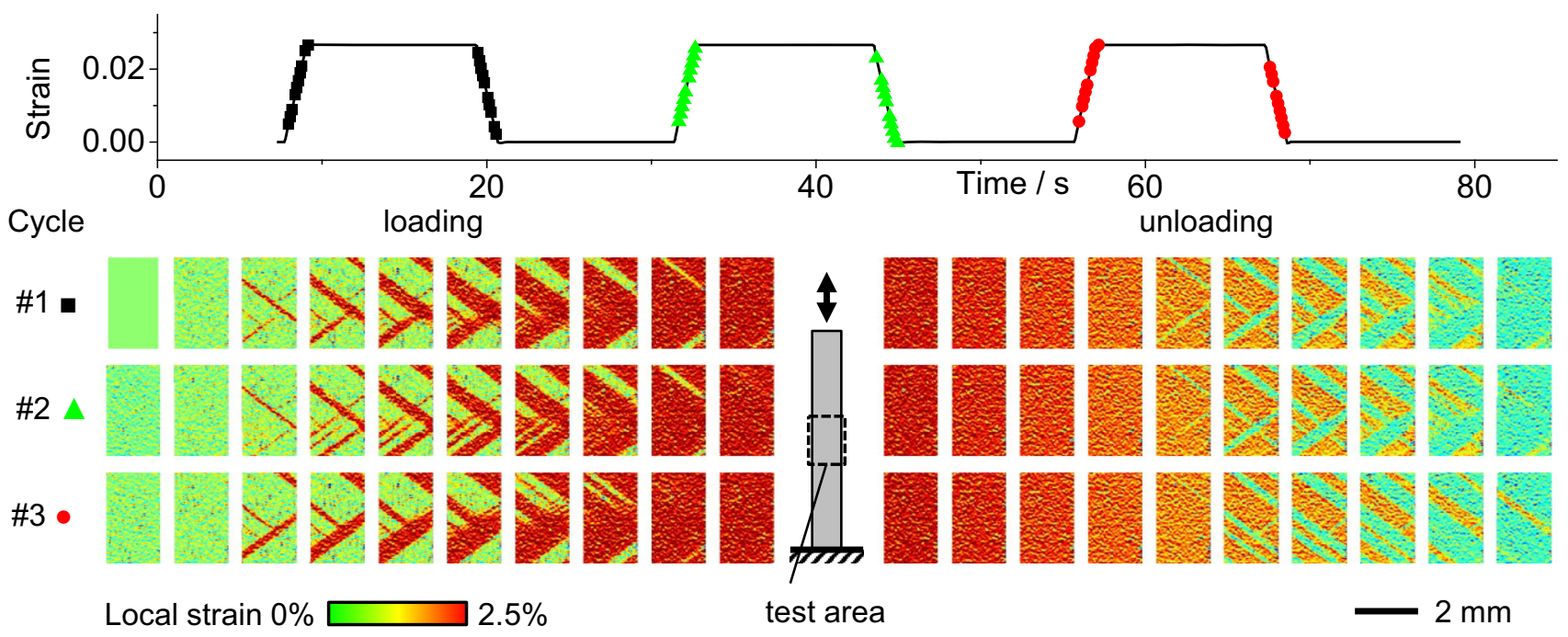

Fig. 6 Time series of local strain maps of a $\mathrm{Ti}_{54.7} \mathrm{Ni}_{30,7} \mathrm{Cu}_{12.3} \mathrm{Co}_{23}$ sample obtained by DIC in a test area $\left(2 \times 3 \mathrm{~mm}^{2}\right)$ from three successive load cycles at a strain rate of $0.02 \mathrm{~s}^{-1}$. The points of image acquisition are marked by symbols in the engineering strain-time diagram above, as well as in the stress-strain characteristic of Fig. 4b

hand show virtually no changes for increasing cycle number except a slight decrease of plateau stress.

The DIC measurements shown in Figs. 5 and 6 reveal the effect of functional fatigue on the mesoscopic length scale. Comparing the strain profiles of the TiNiCuCo sample for successive cycles, a similar pattern of bands is observed (Fig. 6). For the TiNi sample, in contrast, distinct strain bands are only observed in the first few cycles (Fig. 5). The interfaces smear out quickly and eventually vanish completely. After ten cycles, an almost homogeneous strain profile is observed indicating a radical change of the evolution of phase transformation from a localized to a highly delocalized mechanism. A concomitant effect of this change at the mesoscopic scale is the disappearance of the stress plateaus in the macroscopic stress-strain response. As long as transformation proceeds by evolution of strain bands, gradual strain changes are not observed reflecting that the phase transformation is correlated over mesoscale distances. For increasing degradation, this correlation gets lost resulting in a widely dispersed course of phase transformation all over the sample. In the context of elastocaloric cooling, the homogeneous course of transformation and modified hysteresis of trained TiNi samples is per se not a disadvantage. However, the mechanical degradation is accompanied by a degradation of thermal performance, which will be addressed below.

\section{Local Thermal Performance}

Figure $7 \mathrm{a}$ shows a series of local temperature profiles determined by in situ IR thermography during load cycling of a TiNiCuCo sample at various strain rates. Large local strain characteristics of the TiNiCuCo samples on the other 
(a) $\mathrm{d} \varepsilon / \mathrm{d} t=10^{-3} \mathrm{~s}^{-1}, \Delta \mathrm{t}=4 \mathrm{~s}$
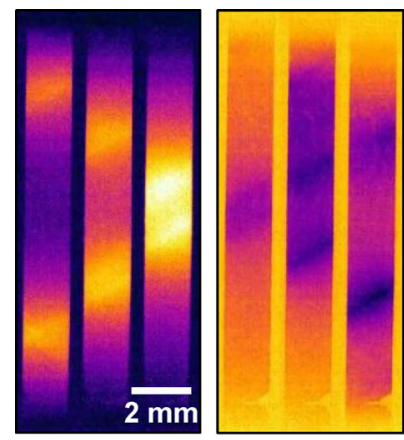

$\mathrm{d} \varepsilon / \mathrm{d} t=10^{-2} \mathrm{~s}^{-1}, \Delta \mathrm{t}=0.16 \mathrm{~s}$

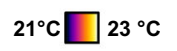

$19.5^{\circ} \mathrm{C} \square 21.5^{\circ} \mathrm{C}$
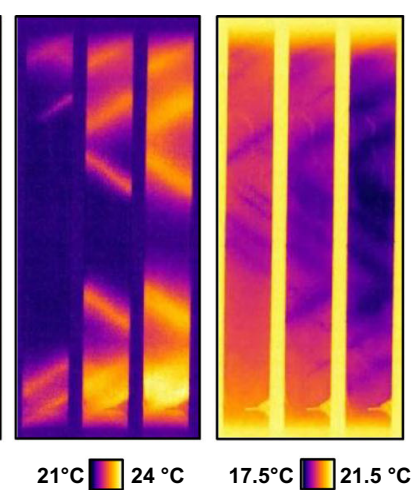

$\mathrm{d} \varepsilon / \mathrm{d} t=10^{-1} \mathrm{~s}^{-1}, \Delta \mathrm{t}=0.02 \mathrm{~s}$

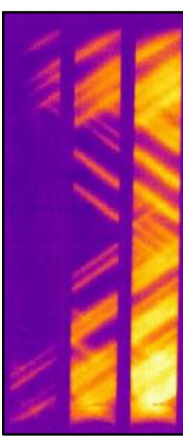

$21^{\circ} \mathrm{C} 25^{\circ} \mathrm{C}$

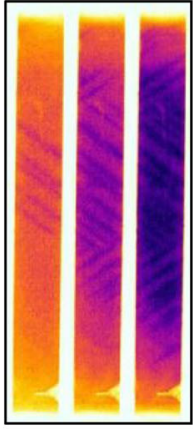

$17.5^{\circ} \mathrm{C} \square 21.5^{\circ} \mathrm{C}$ $\mathrm{d} \varepsilon / \mathrm{d} t=1 \mathrm{~s}^{-1}, \Delta \mathrm{t}=0.01 \mathrm{~s}$

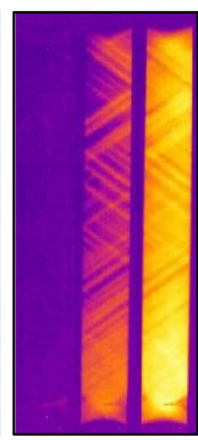

$21^{\circ} \mathrm{C} 27^{\circ} \mathrm{C}$

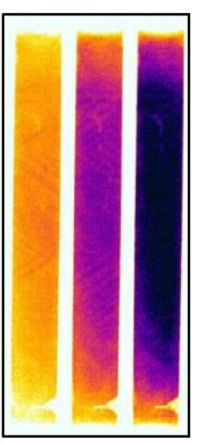

$17.5^{\circ} \mathrm{C} \square 21.5^{\circ} \mathrm{C}$ (b)

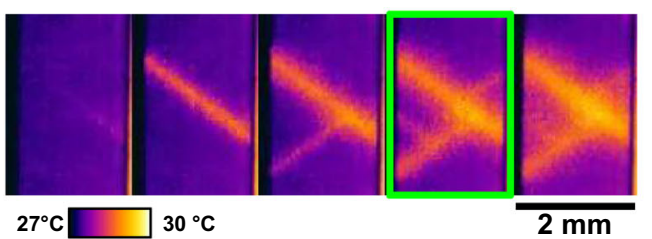

(c)

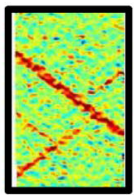

$0 \% \square 5 \%$
Fig. 7 a Series of local temperature profiles obtained by IR thermography during mechanical loading (left) and unloading (right) of a $\mathrm{Ti}_{54.7} \mathrm{Ni}_{30.7} \mathrm{Cu}_{12.3} \mathrm{Co}_{2.3}$ sample at various strain rates. The strain rate $\mathrm{d} \varepsilon / \mathrm{d} t$ and the time interval $\Delta t$ between subsequent images are indicated. b Detailed view shows the formation of temperature bands in a test area of $2 \times 3 \mathrm{~mm}^{2}$ at strain rate $0.02 \mathrm{~s}^{-1}$. c Strain map obtained by DIC corresponding to the IR profile in $\mathbf{b}$ highlighted in green

temperature changes occur during stress-induced martensite forward and reverse transformation originating from the local release and absorption of latent heat as well as heat exchange with the environment. Detailed maps of temperature and strain profiles are depicted in Fig. $7 \mathrm{~b}$ and c highlighting that the formation of temperature and strain bands is strongly correlated [10]. In the following, the temperature profiles are discussed in more detail with respect to their dependence on strain, strain rate, and cycle number.

\section{Strain Dependence}

Formation of hot temperature bands is correlated with the formation of strain bands, once a critical stress value $\sigma_{\text {crit }}$ is exceeded upon loading. Fully transformed regions of oriented martensite show a large temperature increase, while fully reverse transformed regions show a large temperature decrease. The temperature bands are oriented at the same orientation angle with respect to the loading direction as their strain counterparts.

Due to the boundary conditions of sample fixation, temperature bands form at the sample edges near the sample fixation. Tensile loading-induced stress shows a maximum at the sample edges. Thus, the preferred phase transformation in these regions gives rise to the local increase of temperature in the initial phase of tensile loading. Furthermore, martensite formation is stabilized in the regions at the sample edges due to stronger conductive cooling compared to the other regions of the sample.

For increasing strain, temperature bands grow in size due to propagation of phase boundaries and additional temperature bands may form. While keeping the sample in maximum strain state, the temperature decreases due to heat transfer and equalizes with the ambient. For decreasing strain, cold temperature bands form at first in those regions, where the forward transformation has been terminated. Subsequently, cold temperature bands increase in size which is correlated to the increase of the bands of zero strain due to propagation of phase boundaries. Heat transfer leads to a temperature change in neighboring regions and thus blurring of the temperature bands. The propagation of temperature bands from the sample edge toward the sample center has been described by finite element simulation taking the influence of the strain gradient of transformed regions on the neighboring untransformed regions into account [10].

This observation of local thermal performance underlines the results of our DIC measurements that pseudoelastic cycling gives rise to a highly localized course of phase transformation. Resulting temperature profiles may be rather inhomogeneous depending on strain rate and cycle number, which has to be taken into account in the design of elastocaloric cooling devices.

\section{Strain-Rate Dependence}

Figure $7 \mathrm{a}$ demonstrates the strong influence of strain rate on the number of temperature bands evolving during load cycling. At low strain rate, only one strain band forms at each end of the test sample and propagates toward the center. In this quasi-stationary limit, heat conduction is much faster than the movement of phase boundaries. Therefore, the formation of bands is little affected by self- 
heating and essentially governed by the boundary conditions of sample fixation. For increasing strain rate, selfheating and self-cooling occur during loading and unloading, respectively, as the heat transfer can no longer equalize the local release/absorption of latent heat. The resulting accumulation/loss of heat impedes the continuous progression of phase transformation. Consequently, discontinuous formation of many strain bands in different regions is energetically favored compared to continuous progression of an existing strain band. At large strain rate, adiabatic conditions are reached. In this case, a multitude of bands occurs virtually at the same time and the sample temperature can be considered homogeneous after a short time of heat equalization. Similar results have been reported for NiTi wires [22].

This performance is advantageous for applications of elastocaloric cooling. Firstly, the temperature changes become maximal and thus facilitate heat transfer with a heat source or sink. Secondly, large strain rates enable short cycle times and thus high cooling power. The localized character of the phase transformation loses importance, as soon as multiple temperature bands occur at once. The time-dependent temperature change can be treated as approximately homogeneous, which facilitates the simulation of the elastocaloric cooling effect.

\section{Dependence on Cycle Number}

TiNiCuCo film samples show a highly reversible phase transformation, which is reflected on the local scale in the formation of similar patterns of strain bands in successive pseudoelastic cycles (Fig. 6), as well as in the corresponding temperature bands. In contrast, TiNi film samples show prominent temperature and strain bands only during the first few cycles. Due to increase in remanent strain, only a fraction of martensite transforms back into austenite during unloading. Thus, only a fraction of latent heat is absorbed after a number of stress cycles, resulting in a smaller overall temperature change [11]. In conclusion, the mechanical changes due to degradation per se do not diminish cooling efficiency as long as they can be corrected or accounted for by appropriate device design. However, the loss of elastocaloric effect size is a clear disadvantage. Hence, reversibly transforming materials such as the TiNiCuCo films are advantageous for elastocaloric cooling applications.

\section{Elastocaloric Cooling}

The strain-dependent local temperature evolution goes along with a strain-dependent average temperature evolution on the macro scale. Figure 8a shows the time-resolved temperature change determined from measured
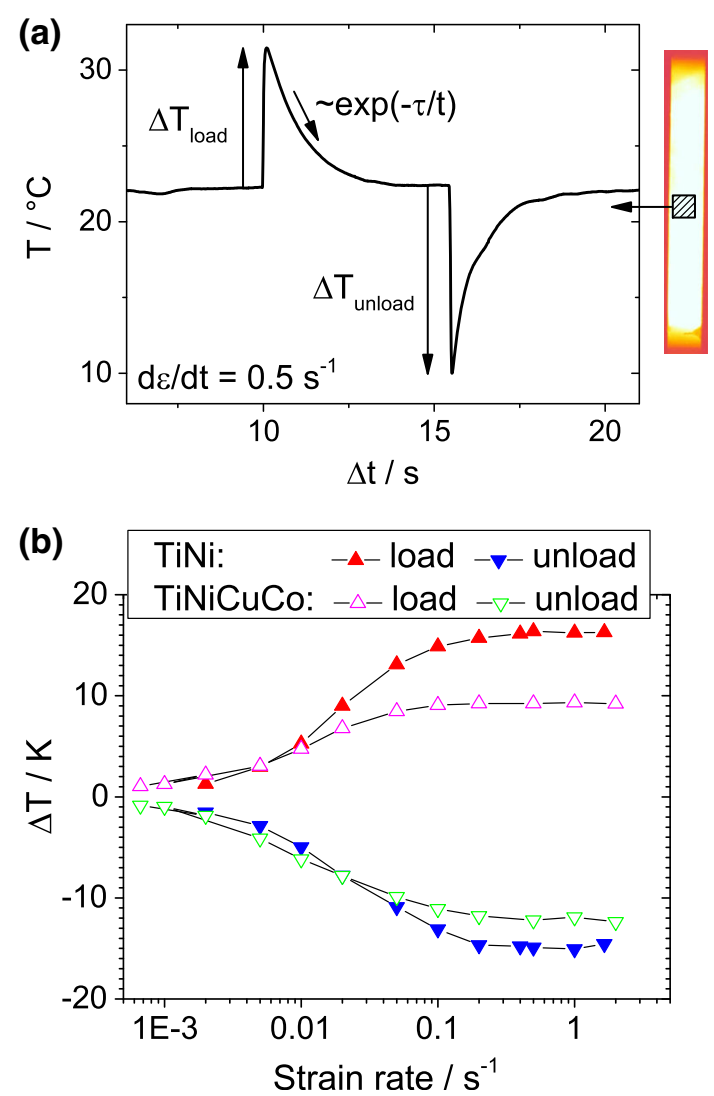

Fig. 8 a Time-dependent evolution of average temperature of a $\mathrm{Ti}_{55} \mathrm{Ni}_{29.6} \mathrm{Cu}_{12.6} \mathrm{Co}_{2.8}$ film sample in a test area of $1 \times 1 \mathrm{~mm}^{2}$ in the sample center as indicated in the inset. $\Delta T_{\text {load }}$ and $\Delta T_{\text {unload }}$ denote the maximum temperature changes upon loading and unloading, respectively. Temperature equalization follows an exponential trend with time constant $\tau$. b Strain-rate dependence of maximum average temperature changes upon loading and unloading for $\mathrm{Ti}_{49.6} \mathrm{Ni}_{50.4}$ and $\mathrm{Ti}_{55} \mathrm{Ni}_{29.6} \mathrm{Cu}_{12.6} \mathrm{Co}_{2.8}$ film samples

temperature profiles by averaging over a test area of $1 \times 1 \mathrm{~mm}^{2}$ in the center of the samples for a strain rate of $0.5 \mathrm{~s}^{-1}$. In this case, $\mathrm{Ti}_{55} \mathrm{Ni}_{29.6} \mathrm{Cu}_{12.6} \mathrm{Co}_{2.8}$ films annealed at $780{ }^{\circ} \mathrm{C}$ are considered showing an improved temperature change $\Delta T$.

After the loading stress exceeds the critical stress for martensitic transformation, the temperature rises proportionally to the strain rate and reaches a strain rate-dependent maximum change $\Delta T$ at the end of transformation. This strain rate dependence is due to the release of heat within a shorter time interval, so that competing heat transfer by heat conduction and convection becomes less effective. At the strain rate of $0.2 \mathrm{~s}^{-1}$, adiabatic conditions are reached. In this case, the strain rate dependence saturates, and we observe a maximum temperature increase of $9 \mathrm{~K}$. While the sample is kept in strained condition at maximum strain for $10 \mathrm{~s}$ the temperature equilibrates with the ambient. The corresponding time constant $\tau$ of heat exchange is about $1 \mathrm{~s}$. Upon unloading, the reverse 
transformation starts instantly. The temperature decrease is again proportional to the strain rate as heat absorption favorably competes with heat transfer. The maximum temperature decrease observed for the strain rate of $0.5 \mathrm{~s}^{-1}$ is about $-12 \mathrm{~K}$. The time constant determined for temperature equalization is about the same for the loading and unloading case.

Figure $8 \mathrm{~b}$ summarizes the results on strain rate-dependent temperature changes for both types of film samples. In both cases, the adiabatic limit is reach at a strain rate of about $0.2 \mathrm{~s}^{-1}$. The highest elastocaloric cooling effect of about $-15 \mathrm{~K}$ is observed in the TiNi films, which is in line with previous results [10]. The TiNiCuCo film samples show a lower elastocaloric effect size. The temperature decrease upon unloading exceeds the temperature increase upon loading, which is quite unexpected and needs further consideration.

\section{Discussion}

The presented investigation shows that the evolution of the elastocaloric effect in the as-received film samples is governed by the formation, propagation, and interaction of strain bands. The evolution of strain bands correlates with temperature bands that form at the phase front of transforming and expanding regions. The abrupt and highly localized course of stress-induced phase transformation observed here is also well known for bulk-like samples with certain geometries including cold-rolled foils of $30-\mu \mathrm{m}$ thickness [12], belt-like samples of 400- $\mu \mathrm{m}$ thickness [27], as well as tubes with wall thickness of $150 \mu \mathrm{m}$ [23]. Formation and propagation of strain bands can be understood by the effect of mesoscale stress and temperature fields on the kinetics of phase transformation [10]. Strain bands may form due to local mesoscale stress concentrations that are generated externally by tensile loading and boundary conditions of sample fixation as well as internally by locally transformed regions. The location of band formation also depends on the local mesoscale temperature profile and load history. Thermodynamically favored regions are the locations of sample fixation, where heat transfer between sample and support structures is enhanced causing stabilization of martensite.

In addition, the strain rate-dependent increase of the number of strain bands can be understood by the competition between band propagation and formation of new bands. At low strain rates, self-heating and self-cooling are negligible. Thus, the local stress field of an already transformed region favors phase transformation in adjacent regions resulting in a continuous propagation of phase fronts from the sample fixation toward the sample center [28]. For increasing strain rate, self-heating and self-cooling gain importance, which has a self-inhibiting effect on the transformation in adjacent regions favoring the formation of new bands and thus a discontinuous progression of phase transformation. Different temperature bands interfere with each other resulting in significant local overheating and undercooling.

Even though major observed phenomena of the elastocaloric effect can be understood in terms of mesoscale stress and temperature fields, the detailed fine structure of strain bands hints at underlying microscale effects. The influence of sample microstructure becomes obvious for the as-received binary TiNi film samples that accumulate large plastic strain during load cycling. The corresponding microstructural defects, in turn, significantly alter the mechanisms involved during evolution of the elastocaloric effect. In particular, we observe that strain bands no longer form in trained TiNi samples. This indicates that phase transformation in different regions is no longer correlated over mesoscale distances, instead they are governed by short-range microscale effects. This raises interesting questions, on which types of defects are involved and how these defects determine the course of phase transformation at the microscale, which is, however, out of scope of the present investigation. The outstanding cyclic stability exhibited by the TiNiCuCo films is currently under investigation. It is attributed to the formation of coherent $\mathrm{Ti}_{2} \mathrm{Cu}$ precipitates showing low misfit with respect to the $\mathrm{B} 2$ parent and B19 phase.

TiNi-based films are of large interest for cooling applications due to the large effect size and the improved heat transfer resulting from high surface-to-volume ratios. Under adiabatic conditions, we observe a large elastocaloric cooling effect associated with the martensitic phase transformation of -16 and $-12 \mathrm{~K}$ for $\mathrm{TiNi}$ and $\mathrm{TiNiCuCo}$ films, respectively. Yet, there is considerable room for improvement, as the maximum possible temperature change estimated from latent heat $L$ and specific heat capacity $c_{\mathrm{p}}$ (Table 1 ) is at least a factor of 2.5 larger, which is in line with maximum reported values for TiNi bulk specimens [6]. Heat generation and absorption are determined by the strain rate. Here, adiabatic heating and cooling occur at a strain rate of $0.2 \mathrm{~s}^{-1}$, which, in the case of TiNiCuCo films, corresponds to time duration of about $140 \mathrm{~ms}$ required for loading and unloading, respectively. Temperature bands equilibrate with the environment through heat conduction and convection within about $1 \mathrm{~s}$, which is at least one order of magnitude shorter compared to bulk specimens $[27,29]$.

In order to assess the potential of TiNi-based films for elastocaloric cooling applications, the material's $\operatorname{COP}(=Q /$ $\Delta W)$ may be considered. Here, this value is estimated from the adiabatic temperature change upon cooling determined by infrared thermography and the work input $\Delta W$ determined 
by numerical integration of the stress-strain characteristic under adiabatic conditions. If the work for unloading is recovered, only the area enclosed by both loading and unloading curves is taken into account. The COP of TiNi films is determined to be about 7.7 after twenty training cycles to allow for stabilization. $\mathrm{Ti}_{55} \mathrm{Ni}_{29.6} \mathrm{Cu}_{12.6} \mathrm{Co}_{2.8}$ films annealed at higher temperatures show a COP of even 15 due to the considerably lower work input.

\section{Conclusions}

Elastocaloric cooling applications require material systems showing large temperature change, short heat transfer times, and high fatigue resistance during load cycling. These aspects are investigated by time-resolved DIC and IR thermography measurements with spatial resolution on the mesoscopic length scale in the micrometer range. The focus is on binary TiNi and quaternary TiNiCuCo films of about of $20-\mu \mathrm{m}$ thickness produced by magnetron sputtering, which strongly differ regarding their phase transformation hysteresis and fatigue behavior.

In the as-received state, the local evolution of the elastocaloric effect is similar in both types of films. IR thermography and DIC measurements reveal a correlated response of strain and temperature bands. Their formation and propagation can be described by the effect of mesoscale stress and temperature fields on the kinetics of phase transformation. The temperature profile is governed by the strain- and strain rate-dependent local release/absorption of heat as well as the heat transfer to the environment. For cooling applications, large strain changes and adiabatic conditions are preferred as this leads to large temperature changes and formation of a multitude of temperature bands, respectively, that quickly equalize to homogeneous temperature profiles. Rapid heat transfer enables the development of film-based elastocaloric cooling devices operating at cycling times in the order of a second. Heat exchange with a solid or liquid medium is expected to be even faster, so that cycling rates of several Hertz seem to be feasible.

Maximum temperature changes in $\mathrm{TiNi}$ and $\mathrm{TiniCuCo}$ film samples in adiabatic condition are -16 and $-12 \mathrm{~K}$, respectively. These results are limited by the accessible latent heat during load cycling and dissipation in the case of TiNi films. Regarding the maximum possible latent heat of the films, an increase by a factor of two seems to be possible.

Reaching the adiabatic limit involves the application of large stress fields, which may lead to functional fatigue or even breakdown. Therefore, it is important to understand the correlation between elastocaloric properties and functional fatigue. On the mesoscopic scale, we find that strain bands disappear within the first few stress cycles and a highly dispersed course of phase transformation in each part of the sample is observed instead. This indicates that fatigue-induced defects hinder the correlation of phase transformation over mesoscale distances. In contrast, TiNiCuCo films showing reproducible stress-strain response retain the local characteristics of the elastocaloric effect. Compared to binary films, they exhibit a reduced temperature change, but an improved material's COP up to 15 due to the lower work required for load cycling.

These results suggest that NiTiCuCo films fulfill the major criteria for cooling applications. Typical examples are in microelectronics, where the increasing power density of state-of-the art processors and electronic components demand for advanced cooling concepts. It is envisioned that SMA film devices may be grouped to arrays or cascaded structures to realize novel-distributed cooling systems.

Acknowledgments The authors gratefully acknowledge funding by the German Science Foundation (DFG) within the priority program SPP1599 (www.ferroiccooling.de).

\section{References}

1. Moya X, Kar-Narayan S, Mathur ND (2014) Caloric materials near ferroic phase transitions. Nat Mat. 13:439-450

2. Fähler S, Rößler UK, Kastner O, Eckert J, Eggeler G, Emmerich H, Entel P, Müller S, Quandt E, Albe K (2012) Caloric effects in ferroic materials: new concepts for cooling. Adv Eng Mater 14(1-2):10-19

3. Cui J, Wu Y, Muehlbauer J, Hwang Y, Radermacher R, Fackler S, Wuttig M, Takeuchi I (2012) Demonstration of high efficiency elastocaloric cooling with large $\Delta \mathrm{T}$ using NiTi wires. Appl Phys Lett 101:73904

4. Mañosa L, Planes A, Vives E, Bonnot E, Romero R (2009) The use of shape-memory alloys for mechanical refrigeration. Funct. Mater. Lett. 2(2):73-78

5. Otubo J, Rigo OD, Coelho AA, Neto CM, Mei PR (2008) The influence of carbon and oxygen content on the martensitic transformation temperatures and enthalpies of NiTi shape memory alloy. Mater Sci Eng, A 481-482:639-642

6. Pieczyska EA, Gadaj SP, Nowacki WK, Tobushi H (2006) Phasetransformation fronts evolution for stress- and strain-controlled tension tests in TiNi shape memory alloy. Exp Mech 46:531-542

7. Mañosa L, Planes A, Acet M (2013) Advanced materials for solid-state refrigeration. J Mater Chem A 1:4925-4936

8. Carmo JP, Silva MF, Ribeiro JF, Wolffenbuttel RF, Alpuim P, Rocha JG, Goncalves LM, Correira JH (2011) Digitally-controlled array of solid-state microcoolers for use in surgery. Microsyst Technol 17:1283-1291

9. El-Ali J, Perch-Nielsen IR, Poulsen CR, Bang DD, Telleman P, Wolff A (2004) Simulation and experimental validation of a SU8 based PCR thermocycler chip with integrated heaters and temperature sensor. Sens Actuators, A 110:3-20

10. Ossmer H, Lambrecht F, Gültig M, Chluba C, Quandt E, Kohl M (2014) Evolution of temperature profiles in TiNi films for elastocaloric cooling. Acta Mater 81:9-20

11. Bechtold C, Chluba C, Lima de Miranda R, Quandt E (2012) High cyclic stability of the elastocaloric effect in sputtered TiNiCu shape memory films. Appl Phys Lett 101:091903 
12. Ossmer H, Miyazaki S, Kohl M (2014) The elastocaloric effect in NiTi-based foils. In: Proceedings of ICOMAT'14, Bilbao, Spain. Materials Today: Proceedings (accepted)

13. Delville R, Kasinathan S, Zhang Z, Van Humbeeck J, James RD, Schryvers D (2010) Transmission electron microscopy study of phase compatibility in low hysteresis shape memory alloys. Philos Mag 90(1-4):177-195

14. Frenzel J, Wieczorek A, Opahle I, Maaß B, Drautz R, Eggeler G (2015) On the effect of alloy composition on martensite start temperatures and latent heats in Ni-Ti-based shape memory alloys. Acta Mater 90:213-231

15. Lima de Miranda R, Zamponi C, Quandt E (2013) Micropatterned freestanding superelastic TiNi films. Adv Eng Mater 15(1-2):66-69

16. Sutton MA, Mingqi C, Peters WH, Chao YJ, McNeill SR (1986) Application of an optimized digital correlation method to planar deformation analysis. Image Vis Comput 4(3):143-150

17. Ling HC, Kaplow R (1980) Phase transitions and shape memory in TiNi. Metall Trans A 11:77-83

18. Miyazaki S, Hashinaga T, Ishida A (1996) Martensitic transformations in sputter-deposited Ti-Ni-Cu shape memory alloy thin films. Thin Solid Films 281-282:364-367

19. Cui J, Chu YS, Famodu OO, Furuya YI, Hattrick-Simpers J, James RD, Ludwig A, Thienhaus S, Wuttig M, Zhang Z, Takeuchi I (2006) Combinatorial search of thermoelastic shapememory alloys with extremely small hysteresis width. Nat Mater 5:286-290

20. Sittner P, Liu Y, Novak V (2005) On the origin of Lüders-like deformation of NiTi shape memory alloys. J Mech Phys Solids 53:1719-1746
21. Shaw JA, Kyriakides S (1997) On the nucleation and propagation of phase transformation fronts in a NiTi alloy. Acta Mater 45(2):683-700

22. Zurbitu J, Kustov S, Zabaleta A, Cesari E, Aurrekoetxea J (2010) In: Cismasiu C (ed) Thermo-mechanical behaviour of NiTi at impact, shape memory alloys. InTech, Vienna

23. Sun QP, Li ZQ (2002) Phase transformation in superelastic NiTi polycrystalline micro-tubes under tension and torsion-from localization to homogeneous deformation. Int J Solids Struct 39(13-14):3797-3809

24. Schlosser P, Louche H, Favier D, Orgéas L (2007) Image processing to estimate the heat sources related to phase transformations during tensile tests of NiTi tubes. Strain 42:260-271

25. He YJ, Sun QP (2011) Rate-dependent damping capacity of NiTi shape memory alloy. Solid State Phenom 172-174:37-42

26. He YJ, Sun QP (2011) On non-monotonic rate dependence of stress hysteresis of superelastic shape memory alloy bars. Int $\mathbf{J}$ Solids Struct 48:1688-1695

27. Pieczyska E (2010) Activity of stress-induced martensite transformation in TiNi shape memory alloy studied by infrared technique. J Mod Opt 57(18):1700-1707

28. Shaw JA, Kyriakides S (1995) Thermomechanical aspects of NiTi. J Mech Phys Solids 43(8):1243-1281

29. Gschneidner KA Jr, Pecharsky VK, Tsokol AO (2005) Recent developments in magnetocaloric materials. Rep Prog Phys 68:1479-1539 